\title{
EVALUACIÓN DEL CONOCIMIENTO DEL PROGRAMA PREESCOLAR DE SALUD BUCAL EN PARVULARIAS, MEDIANTE UNA ENCUESTA
}

\section{KNOWLEDGE ASSESSMENT OF PRESCHOOL ORAL HEALTH PROGRAM IN NURSERY SCHOOLS, THROUGH A SURVEY}

\section{Chang Silvana ${ }^{1}$, Quiroz Mariela. ${ }^{2}$}

\section{RESUMEN}

Objetivo: Diseñar y determinar un instrumento que evalúe el conocimiento de las educadoras de párvulo respecto a la salud oral de los niños en Valparaíso.

Métodos: Estudio de serie de casos, transversal. Se realizó una encuesta de 23 preguntas que se basaron en los contenidos del "Programa de promoción y prevención en salud bucal para niños y niñas preescolares, MINSAL, 2007" aplicada a 244 parvularias.

Resultados: La encuesta presentó un Alpha de Cronbach de 0,84. Se obtuvo una media de nota 3,5 (IC $95 \%$ : 3.4-3.6) según la escala del 1 al 7 , que representa el nivel de conocimiento de las parvularias, en donde el área mejor calificada fue la alimentación.

Conclusiones: Las parvularias presentan conocimiento deficiente en relación al programa de salud bucodentaria, por lo que es necesaria una capacitación en relación a esta temática.

\section{ABSTRACT}

Objective: To design and determine an instrument that evaluates the knowledge of nursery educators regarding the oral health of children in Valparaíso.

Methods: Case series, cross-sectional study. A survey of 23 questions was conducted based on the contents of the "Oral health promotion and prevention program for preschool children of MINSAL 2007" applied to 244 nursery schools.

Results: The survey presented a Cronbach Alpha of 0.84 . A 3.5 grade point average (95\% Cl: 3.4-3.6) was obtained according to the scale from 1 to 7 , which represents the level of knowledge of nursery schools, where the best qualified area was Nutrition.

Conclusions: Nursery schools have poor knowledge in relation to oral health program of preschool children, so training in relation to this topic is necessary.
1. Centro de Salud Familiar Iris Veliz Hume, Arica, Chile

2. Universidad de Valparaíso, Escuela de Odontología, Valparaíso, Chile.

Correspondencia:

Silvana Chang

Correo electrónico:

changsilvana@gmail.com

\section{PALABRAS CLAVES:}

Encuesta; conocimiento; parvularias; salud bucal; estudio de validación.

\section{KEYWORDS:}

Survey; knowledge; preschool; oral health; validation study. 


\section{INTRODUCCIÓN}

En Chile las patologías bucales presentan alta prevalencia y severidad afectando la calidad de vida de los niños y sus familias. Hoy en día cada vez son más las familias que le entregan el cuidado de sus hijos a centros educacionales externos. En Chile el 19\% de los menores de tres años y el $74 \%$ de los niños entre cuatro y cinco años asiste a establecimientos de educación parvularia. ${ }^{1}$

El Ministerio de Salud de Chile (MINSAL) diseñó un manual educativo el año 2007, en el marco del "Programa de promoción y prevención en salud bucal para niños menores de 6 años", con el objetivo de entregar un instrumento para las acciones de capacitación para el personal a cargo de los niños, sin embargo, han pasado más de 10 años desde su creación y no es válido usarlo por los avances que han generado nuevos conocimientos. Por lo anterior dicho se hace necesario evaluar a las parvularias con un instrumento actualizado que nos indique cuánto saben de salud oral.

Es importante determinar el grado de conocimiento en salud oral que poseen las educadoras de párvulo, debido a que esto indicará si están capacitadas para entregar educación en salud bucal a los preescolares. Además, al evaluarlas podremos ver las áreas débiles que requieren refuerzo por el profesional de salud.

El objetivo del estudio es diseñar y determinar un instrumento para medir el nivel de conocimiento en salud bucal de las educadoras de párvulo y técnicos en educación parvularia de los jardines infantiles, según los contenidos del "Programa de promoción y prevención en salud bucal para niños y niñas preescolares, MINSAL, 2007”.

\section{MATERIAL Y MÉTODOS}

Se realizó un diseño de serie de casos, transversal descriptivo. La población a estudiar correspondió a 244 parvularias de Valparaíso. A través de una encuesta de 23 preguntas; basadas en los contenidos del "Programa de promoción y prevención en salud bucal para niños y niñas preescolares, MINSAL 2007"; se recogieron las siguientes variables: edad en escala discreta, nivel educacional (básico, medio, técnico medio o superior, especialización profesional o universitario), jornada laboral (horas al día), niños a cargo (menor a 5, 6 a 10, o más), institución a la que pertenece el jardín (Junta Nacional de Jardines Infantiles (JUNJI), Integra, Vía Transferencia de Fondos (VTF) y privados). También se consideró puntaje y nota ( 1 al 7 PREMA 60\%) según conocimiento.

Para la elaboración de la encuesta se revisaron instrumentos similares disponibles en la literatura médica, a través de una búsqueda en bases de datos internacionales con los descriptores: "surveys and questionnaires", "healthknowledge, attitudes, practice", "caregivers/psychology", "childdaycare centers", "healtheducation, dental", "oralhealth"; "estudios de validación"; y el manual educativo "Programa de promoción y prevención en salud bucal para niños y niñas preescolares, MINSAL, 2007”.

Se formularon las preguntas utilizando los criterios de redacción, según Arribas 2004. ${ }^{2}$ Las cuales evaluaban conocimiento sobre los contenidos del programa anteriormente nombrado. Se establecieron preguntas asociadas a la alimentación, higiene oral, aspectos clínicos odontológicos (como manifestación clínica de caries, erupción dentición temporal, primeros auxilios en traumatismo dentoalveolar (TDA) y programas odontológicos infantiles en Chile). Finalizando con preguntas relacionadas a malos hábitos orales.

Una vez redactados las preguntas, éstas se agruparon por contenidos. Se realizó un constructo de 7 preguntas sobre información sociodemográfica, y 23 preguntas de conocimiento, obteniendo la versión 1 de la encuesta.

Se evaluó la validez de contenido de la encuesta, aplicando la versión 1 de la misma (Pretest), en 11 especialistas en odontopediatría, 3 voluntarios profesionales no afines al área de la salud y 15 educadoras de párvulo. Las preguntas se evaluaron según: pregunta y extensión adecuada, enunciado correcto y comprensible, correcta 
categorización de las respuestas, presencia de rechazo hacia estas, ordenamiento interno lógico y duración aceptable de la encuesta.

Se analizaron las sugerencias de modificación de las personas que respondieron el Pretest. Realizando la versión 2 de la encuesta, la cual fue analizada por 11 especialistas en odontopediatría, quienes evaluaron la formulación de las preguntas y respuestas. Se indicó marcar con una "X" en el recuadro donde se estimó más adecuada su puntuación según la escala Likert. ${ }^{3}$

La encuesta (versión 2), se efectúo en enero del 2019 en educadoras de párvulo y técnicos en educación parvularia de jardines infantiles de Valparaíso. Las encuestadas se seleccionaron por muestreo no probabilístico por conveniencia. Se utilizaron los siguientes criterios de inclusión de: educadoras de párvulo pertenecientes a salas cuna y jardines infantiles particulares de la comuna de Valparaíso con autorización normativa de JUNJI o empadronamiento actualizado a abril del 2018, además que estos estuvieran operativos a junio de 2018, administrados directamente por JUNJI, VTF y convenio. 42 jardines infantiles cumplían con los criterios de inclusión.

Y se excluyeron los jardines que: entre el 20162018 fueron capacitados por residentes de odontopediatría de la Universidad de Valparaíso (UV) (en total 2), aquellos no autorizados por la administración directa de JUNJI (en total 5), los que no respondieron las llamadas o correos (en total 14), y los que no estaban interesados en participar (en total 12).

A cada encuestada se les hizo firmar un consentimiento informado. La encuesta se realizó de forma anónima, autoaplicada y presencial. En donde se evaluó si contestaba correcta o incorrectamente, las preguntas omitidas fueron consideradas como erróneas.

Se le entregó un número correlativo a cada encuestada de modo que pudieran acceder al resultado de las encuestas.

Los análisis estadísticos utilizados fueron Alpha de Cronbach, para verificar la validez de contenido, y Teoría de respuesta al ítem (IC: $95 \%$ ), el cual analizó las preguntas de la encuesta identificando su valor significativo con respecto al poder discriminante del conocimiento y el grado de dificultad.

Los análisis de los datos se realizaron a través del Software: STATA, Microsoft Excel 2016 y RCran 3.5.1 versión 64x.

El estudio fue analizado por el Comité ÉticoCientífico de la Facultad de odontología de la UV, el 23 de octubre del 2018.

\section{RESULTADOS}

La versión 2 de la encuesta obtuvo un Alpha de Cronbach de 0,84 , lo que indicó una consistencia interna "buena". En esta encuesta, se reformularon las preguntas y respuestas del ítem de conocimiento de la versión 1 , de manera que se eliminó la alternativa "no responde por falta de compresión de la pregunta", además las preguntas se hicieron más específicas, atingentes al tema a evaluar y se usaron palabras más simples, evitando tecnicismos propios de la profesión.

En un principio se seleccionaron 42 establecimientos, de los cuales al aplicar los criterios de exclusión se descartaron 33, quedando 15 jardines infantiles correspondientes al $35,7 \%$ del total de la comuna, de los cuales se obtuvieron 244 encuestadas.

En relación al nivel educacional era principalmente técnico nivel superior (34\%), seguido del universitario $(31,2 \%)$ y del técnico nivel medio $(29,1 \%)$.

Por otra parte, sólo un $32 \%$ de las educadoras parvularias ha declarado haber participado en talleres de capacitación en salud oral.

Respecto al trabajo de las educadoras, el recinto parvulario más frecuente fueron los asociados a la institución JUNJI con un 70\%, en comparación al VTF (20\%) y el privado (10\%). El $82 \%$ de las parvularias manifestó tener más de 10 niños a su cargo.

En la segunda parte de la encuesta se obtuvo que 
las parvularias poseen un $75,41 \%$ de conocimientos con respecto al área de alimentación, teniendo su principal fortaleza en la pregunta relacionada con la alimentación saludable (Tabla I).

Con respecto a los hábitos del niño las parvularias poseen un $46,52 \%$ de conocimiento, teniendo mayor déficit en relación a la pregunta de "la situación que ocasiona mayor malformación en la boca" y el uso del chupete (Tabla II).

En relación a la higiene oral, las parvularias poseen un $49,13 \%$ de conocimiento, siendo las preguntas con menor porcentaje de aciertos, la de "factor que aumenta el riesgo de caries" y la relacionada con la concentración de flúor mínima (Tabla III).

\section{Tabla I: Resultados de alimentación}

\begin{tabular}{|c|c|c|c|c|}
\hline $\begin{array}{l}\mathrm{N}^{\circ} \text { de la } \\
\text { pregunta }\end{array}$ & $\begin{array}{l}\text { Variable de la } \\
\text { Pregunta }\end{array}$ & Alternativas & $\mathrm{N}^{\circ}$ & $\%$ \\
\hline \multirow[t]{2}{*}{2} & \multirow{2}{*}{$\begin{array}{l}\text { Incorporar } \\
\text { alimentos } \\
\text { picados en la } \\
\text { alimentación }\end{array}$} & $\begin{array}{l}\text { Salida de los primeros molares, } \\
\text { desarrollo de la masticación y la } \\
\text { deglución del bebé }\end{array}$ & 149 & 61 \\
\hline & & Otras alternativas & 95 & 39 \\
\hline \multirow{2}{*}{3} & \multirow{2}{*}{$\begin{array}{l}\text { Alimentos } \\
\text { cariogénicos }\end{array}$} & Alimentos ricos en harinas refinadas & 167 & 68 \\
\hline & & Otras alternativas & 77 & 32 \\
\hline \multirow{2}{*}{4} & \multirow{2}{*}{$\begin{array}{l}\text { Alimentación } \\
\text { saludable }\end{array}$} & Frutas, verduras frescas & 238 & 97,5 \\
\hline & & Otras alternativas & 6 & 2,5 \\
\hline \multirow{2}{*}{5} & \multirow{2}{*}{$\begin{array}{l}\text { Principal fuente } \\
\text { de flúor }\end{array}$} & Agua potable de la llave & 182 & 75 \\
\hline & & Otras alternativas & 62 & 25,4 \\
\hline
\end{tabular}

Tabla II: Resultados sobre hábitos del niño

\begin{tabular}{|c|c|c|c|c|}
\hline $\begin{array}{l}\mathrm{N}^{0} \text { de la } \\
\text { pregunta }\end{array}$ & $\begin{array}{l}\text { Variable de la } \\
\text { Pregunta }\end{array}$ & Alternativas & $\mathrm{N}^{\mathrm{o}}$ & $\%$ \\
\hline \multirow{2}{*}{1} & \multirow{2}{*}{$\begin{array}{l}\text { Lactancia materna } \\
\text { exclusiva }\end{array}$} & 6 meses & 156 & 64 \\
\hline & & Otras alternativas & 88 & 36 \\
\hline \multirow[b]{2}{*}{17} & \multirow{2}{*}{$\begin{array}{l}\text { Objeto o situación } \\
\text { que ocasiona } \\
\text { mayor } \\
\text { malformación en } \\
\text { la boca del niño }\end{array}$} & Chuparse el dedo & 46 & 19 \\
\hline & & Otras alternativas & 198 & 81 \\
\hline \multirow{2}{*}{18} & \multirow{2}{*}{ Uso de mamadera } & $\begin{array}{l}\text { Aumentan el riesgo de tener caries } \\
\text { después del año de edad cuando toman } \\
\text { mamadera durante la noche }\end{array}$ & 191 & 78,3 \\
\hline & & Otras alternativas & 53 & 22 \\
\hline \multirow{2}{*}{19} & \multirow{2}{*}{ Uso de chupete } & $\begin{array}{l}\text { Se recomienda eliminar durante los } 2 \text { y } \\
3 \text { años }\end{array}$ & 61 & 25 \\
\hline & & Otras alternativas & 183 & 75 \\
\hline
\end{tabular}




\section{Tabla III: Resultados sobre higiene oral}

\begin{tabular}{|c|c|c|c|c|}
\hline $\begin{array}{l}\mathrm{N}^{0} \text { de la } \\
\text { pregunta }\end{array}$ & $\begin{array}{l}\text { Variable de la } \\
\text { Pregunta }\end{array}$ & Alternativas & $\mathrm{N}^{\mathrm{o}}$ & $\%$ \\
\hline \multirow{2}{*}{10} & \multirow{2}{*}{$\begin{array}{l}\text { Factor que } \\
\text { aumenta el riesgo } \\
\text { de caries }\end{array}$} & Cuando el niño se cepilla 1 vez al día & 30 & 12,3 \\
\hline & & Otras alternativas & 214 & 88 \\
\hline \multirow{2}{*}{9} & \multirow{2}{*}{$\begin{array}{l}\text { Método más } \\
\text { efectivo del } \\
\text { cuidado de salud } \\
\text { bucal }\end{array}$} & $\begin{array}{l}\text { Cepillado frecuente con pasta dental con } \\
\text { flúor }\end{array}$ & 179 & 73,4 \\
\hline & & Otras alternativas & 65 & 27 \\
\hline \multirow[t]{2}{*}{10} & \multirow{2}{*}{$\begin{array}{l}\text { Características del } \\
\text { cepillo }\end{array}$} & $\begin{array}{l}\text { Cabeza pequeña, cerdas suaves y } \\
\text { cambiarse idealmente cada } 3 \text { meses }\end{array}$ & 215 & 88,1 \\
\hline & & Otras alternativas & 29 & 12 \\
\hline \multirow[t]{2}{*}{11} & \multirow{2}{*}{$\begin{array}{l}\text { Edad de inicio de } \\
\text { limpieza bucal del } \\
\text { bebé }\end{array}$} & $\begin{array}{l}\text { Antes que los dientes aparezcan en boca } \\
\text { (erupcionen) }\end{array}$ & 150 & 61 \\
\hline & & Otras alternativas & 94 & 39 \\
\hline \multirow{2}{*}{12} & \multirow{2}{*}{$\begin{array}{l}\text { Edad } \\
\text { recomendable } \\
\text { para iniciar el } \\
\text { cepillado dental } \\
\text { con pasta de } \\
\text { dientes con flúor }\end{array}$} & Desde que aparece el primer diente & 54 & 22,1 \\
\hline & & Otras alternativas & 190 & 78 \\
\hline \multirow{2}{*}{13} & \multirow{2}{*}{$\begin{array}{l}\text { Concentración } \\
\text { mínima de flúor }\end{array}$} & 1.000 ppm de flúor & 32 & 13 \\
\hline & & Otras alternativas & 212 & 87 \\
\hline \multirow{2}{*}{14} & \multirow{2}{*}{$\begin{array}{l}\text { Cantidad de pasta } \\
\text { dental en menores } \\
\text { de } 2 \text { años }\end{array}$} & Sólo la pinta del cepillo o raspado & 46 & 19 \\
\hline & & Otras alternativas & 198 & 81,1 \\
\hline \multirow{2}{*}{15} & \multirow{2}{*}{$\begin{array}{l}\text { Cantidad de pasta } \\
\text { dental en mayores } \\
\text { de } 2 \text { años }\end{array}$} & Tamaño de arveja & 186 & 76,2 \\
\hline & & Otras alternativas & 58 & 24 \\
\hline \multirow{2}{*}{16} & \multirow{2}{*}{$\begin{array}{l}\text { Razón de por qué } \\
\text { el cepillado debe } \\
\text { ser supervisado } \\
\text { por un adulto }\end{array}$} & $\begin{array}{l}\text { Para corregir y estimular la técnica y el } \\
\text { hábito de cepillado }\end{array}$ & 187 & 77 \\
\hline & & Otras alternativas & 57 & 23,4 \\
\hline
\end{tabular}

Con respecto a las preguntas sobre aspectos clínicos, se obtuvo que un $39,97 \%$ de parvularias tiene conocimientos sobre este tema, teniendo un déficit principalmente en los primeros auxilios a realizar frente a una avulsión de diente temporal y cuándo es el término de aparición de dientes temporales (Tabla IV).

En el análisis descriptivo se encontró que las preguntas p3, p10, 05, p18, p21, p11 y p13, tenían mayor probabilidad de ser contestadas en virtud del conocimiento, ya poseían un valor sobre 0.1 (Figura 1).
$\mathrm{Al}$ analizar el puntaje obtenido por las parvularias este tuvo una media de 11,46 (IC 95\%: 11.11 11.81), lo que nos da una nota según conocimiento de 3,52 (IC 95\%: 3.44 - 3.60) con un PREMA $60 \%$, ya que solo contestaron correctamente un 50\% de las preguntas (IC 95\%: $0.48-0.51)$.

Se obtuvieron diferencias estadísticamente significativas al comparar los resultados de las parvularias que recibieron capacitación en salud oral, con las que no. Las primeras obtuvieron una media de puntaje de 12,33 (IC 95\%: 11.82 - 
12.84), que se traduce en una nota 3,71 (IC 95\%: 3.59 - 3.84) con un 54\% de preguntas correctas (IC 95\%: 0.51 - 0.56). Mientras que las parvularias que no recibieron capacitación obtuvieron una media de puntaje de 10,91 (IC 95\%: 10.44 - 11.38), obteniendo una nota 3,4 (IC 95\%: 3.29 - 3.51), con un $47 \%$ de preguntas correctas (IC 95\%: 0.45 - 0.49).

Al comparar los resultados de las parvularias según su nivel educacional y según la institución a la que pertenecían, no se encontraron resultados estadísticamente significativos.

\section{Tabla IV: Resultados sobre aspectos clínicos odontológicos}

\begin{tabular}{|c|c|c|c|c|}
\hline $\begin{array}{l}\mathrm{N}^{0} \text { de la } \\
\text { pregunta }\end{array}$ & Variable de la Pregunta & Alternativas & $\mathrm{N}^{\mathrm{o}}$ & $\%$ \\
\hline \multirow{2}{*}{ r. -8} & \multirow{2}{*}{$\begin{array}{l}\text { Término de aparición } \\
\text { de dientes temporales }\end{array}$} & $\begin{array}{l}\text { Los dientes temporales terminan de } \\
\text { aparecer con seguridad a los } 3 \text { años }\end{array}$ & 75 & 31 \\
\hline & & Otras alternativas & 169 & 69,2 \\
\hline \multirow{2}{*}{7} & \multirow{2}{*}{$\begin{array}{l}\text { Primer signo de caries } \\
\text { dental }\end{array}$} & Mancha blanca & 80 & 33 \\
\hline & & Otras alternativas & 20 & 67,2 \\
\hline \multirow{2}{*}{20} & \multirow{2}{*}{$\begin{array}{l}\text { Respecto a la edad en } \\
\text { la que se debe llevar a } \\
\text { los niños/niñas por } \\
\text { primera vez al dentista }\end{array}$} & A los 6 meses & 89 & 36,5 \\
\hline & & Otras alternativas & 155 & 63,5 \\
\hline \multirow[t]{2}{*}{21} & \multirow{2}{*}{$\begin{array}{l}\text { Respecto al TDA en } \\
\text { dientes temporales }\end{array}$} & $\begin{array}{l}\text { Afecta al diente y estructuras que lo } \\
\text { rodean (encía y hueso) }\end{array}$ & 106 & 43,4 \\
\hline & & Otras alternativas & 138 & 56,5 \\
\hline \multirow[t]{2}{*}{22} & \multirow{2}{*}{$\begin{array}{l}\text { Respecto a los } \\
\text { primeros auxilios de } \\
\text { avulsión de un diente } \\
\text { temporal }\end{array}$} & $\begin{array}{l}\text { Limpiar las heridas de la boca y } \\
\text { acudir rápidamente al dentista }\end{array}$ & 76 & 31,1 \\
\hline & & Otras alternativas & 169 & 69 \\
\hline \multirow[t]{2}{*}{23} & \multirow[t]{2}{*}{$\begin{array}{l}\text { Respecto a los } \\
\text { Programas } \\
\text { Odontológicos } \\
\text { disponibles para los } \\
\text { niños y niñas del } \\
\text { CESFAM }\end{array}$} & $\begin{array}{ll} & \text { Atención de Urgencia } \\
& \text { Odontológica Ambulatoria } \\
\text { - } & \text { Programa CERO } \\
\text { - } & \text { Programa Odontológico para } \\
& \text { niños(as) de } 6 \text { años } \\
\end{array}$ & 102 & 42 \\
\hline & & Otras alternativas & 142 & 58,1 \\
\hline
\end{tabular}

Tabla V: Clasificación de las preguntas según grado discriminante de conocimiento y grado de dificultad

\begin{tabular}{|c|c|c|c|}
\hline Preguntas № & Grado Discriminante & Grado Dificultad & Valor de la Pregunta \\
\hline $\begin{array}{c}1,3,5,7,10,11,13, \\
15,16 \text { y } 18\end{array}$ & Significativo & Significativo & Buena \\
\hline $\begin{array}{c}2,4,8,12,14,19, \\
20 \text { y } 22\end{array}$ & Dudoso & Dudoso & Mala \\
\hline 9 & Dudoso & Significativo & Regular-mala \\
\hline 21 y 23 & Significativo & Dudoso & Regular-mala \\
\hline
\end{tabular}


Figura 1: Gráfico de descripción de preguntas, según su grado de información vs coeficiente de rasgo latente.

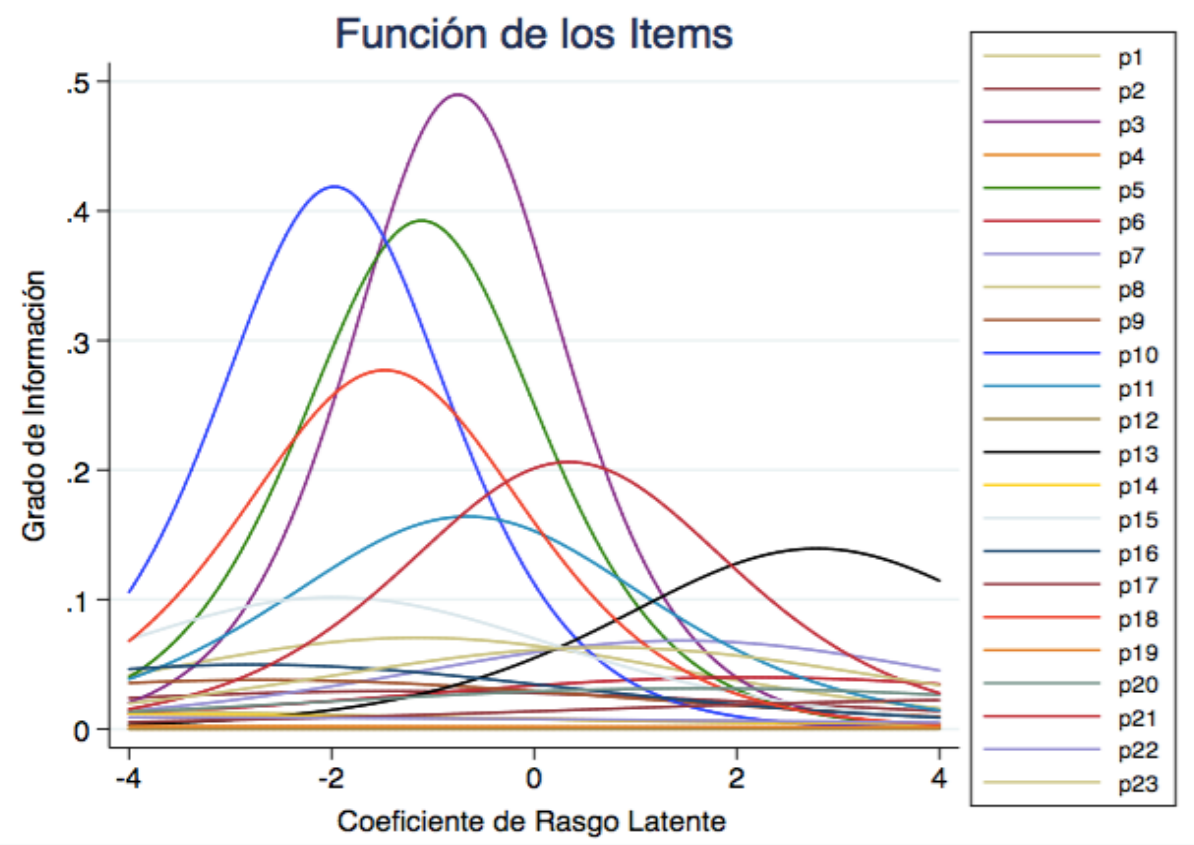

\section{DISCUSIÓN}

Los principales hallazgos del estudio fueron que las parvularias tienen mayor conocimiento en cuanto al área de alimentación, siendo el resultado sobre el $50 \%$. Mientras que en las otras áreas de higiene oral, hábitos del niño y aspectos clínicos odontológicos, las respuestas correctas fueron menos del $50 \%$, esto se puede deber a que para obtener el título de educadora las mallas curriculares incluyen un ramo de nutrición en el niño.,

Existe un déficit en relación a los conocimientos sobre traumatismo dentoalveolar, lo cual es negativo ya que la mayor frecuencia de traumatismo dentoalveolar ocurre en colegios y jardines infantiles ${ }^{6}$, por lo tanto las parvularias deberían tener los conocimientos básicos para prestar los primeros auxilios.

Las parvularias tienen un $46,52 \%$ de conocimiento respecto a los malos hábitos orales, lo cual es preocupante puesto que los malos hábitos tienen relación con las anomalías dentomaxilares, un estudio realizado por Machado Sc el 2018 en niños portugueses, demostró que los niños con hábitos de succión no nutritivos, como lo sería la utilización del chupete, produce alta prevalencia de una clase II de Angle lo que generaría a largo plazo la necesidad de un tratamiento ortodóncico con sus costos e invasión correspondiente. ${ }^{7}$

En relación a conocimientos de higiene oral, se encontró un déficit en relación a la concentración de los fluoruros, lo cual es relevante porque los niños pasan varias horas en los jardines y es ahí donde deben realizar el cepillado dental, además la pasta dental, es uno de los elementos que está incluido en la lista de útiles personales que debe llevar el párvulo al Jardín Infantil; y las educadoras deben supervisar que la pasta dental entregada sea la correcta. Por esto es necesario que las educadoras estén informadas, ya que influye directamente en la prevención de caries a futuro. El MINSAL relata en la Norma de uso de fluoruros en la prevención odontológica, que en el grupo de menores de 6 años, el uso de pastas con concentraciones de flúor de 1000 a 1500 ppm tienen mayor efecto protector comparado con las pastas de baja concentración de flúor (menor a 600 ppm), observándose diferencias estadísticamente significativas. Al comparar las pastas de menos de 600 ppm con placebos, no 
existe evidencia consistente que muestre un efecto protector de caries de las pastas de baja concentración de flúor. ${ }^{8}$

Como en general los conocimientos del programa del MINSAL son bajos, esto se relaciona con el bajo porcentaje de educadoras parvularias que han participado en talleres de capacitación en salud oral. Según un estudio realizado en Chile, que evaluaba los conocimientos en salud bucal en estudiantes de último año de educación parvularia, las futuras educadoras tienen poco conocimiento en salud oral, y no parecen haber adquirido ningún conocimiento nuevo y significativo sobre el tema durante su tiempo en la universidad. ${ }^{9}$ Es por ello que es importante realizar una capacitación posterior.

Las parvularias al pasar gran parte del tiempo con los niños, son importantes para pesquisar posibles problemas odontológicos si tuvieran mayores conocimientos en esta área, por ende, podrían ser colaboradoras en prevención, detección temprana y comunicación a los padres. El estudio de Mishra A y cols. establece una relación directa entre conocimiento y beneficio, ya que cuando los padres tienen una correcta educación en salud oral, se condice con el estado bucal de los niños. ${ }^{10}$

La encuesta presenta validez de contenido con un Alpha de Cronbach bueno. Sin embargo, la muestra no fue aleatoria ni representativa, ya que solo incluía un tercio de la totalidad de los jardines de la comuna de Valparaíso, por eso se sugiere que se utilicen las preguntas catalogadas como buenas y se realice en una muestra representativa.

Podemos usar este cuestionario como base, para que así se tenga un instrumento confiable que nos ayude, en los años venideros, a evaluar a las educadoras y así saber en qué áreas se debe hacer hincapié. De esta forma se podrá implementar alguna medida de capacitación en cuanto a salud bucodentaria, lo cual ayudaría a prevenir de forma temprana alteraciones $\mathrm{o}$ patologías orales en los niños.

\section{CONCLUSIÓN}

Se diseñó un instrumento para evaluar el conocimiento de las educadoras de párvulos sobre el "Programa de promoción y prevención en salud bucal para niños y niñas preescolares, MINSAL, 2007', el cual presentó validez de contenido.

Las educadoras de párvulo son parte esencial en el desarrollo de los niños, y si ellas saben sobre la educación bucodentaria pueden ser factor clave en la prevención del desarrollo de enfermedades bucales a futuro.

\section{CONFLICTOS DE INTERÈS}

Sin conflictos de interés

\section{REFERENCIAS}

[1] Ministerio desarrollo social. Chile, Ministerio de Planificación y Cooperación. 2010. Disponible http://www.ministeriodesarrollosocial.gob. cl/casen2009/

[2] Arribas, M.. Diseño y validación de cuestionarios. Matronas profesión. 2004; 5(17), 23-29.

[3] Matas, A. (2018). Diseño del formato de escalas tipo Likert: un estado de la cuestión. Revista Electrónica de Investigación Educativa, 20(1), 38-47.

[4] ICEL. Chile: ICEL, 2008 [Citado 29 jul 2019]. Disponible en: http://www.icel.cl/tecnico-eneducacion-parvularia/

[5] Universidad de Chile. Chile. 2019 [Citado 29 jul 2019]. Disponible en: http://www.uchile.cl/carreras/105700/pedagog ia-en-educacion-parvularia

[6] Ávila, C., Cueto, A., \& González, J. Caracterización del traumatismo dentoalveolar que afecta a los tejidos de soporte en dientes temporales. Scielo. 2012; 6(2), 157-161.

[7] Machado, S., Manzanares, M., Ferreira, J., Ferreira, J., Rompante, P., \& Ustrell, J. Sample of non-nutritive sucking habits (pacifier and digit) in portuguese children and its relation with the molar classes of Angle. Journal of clinical and experimental dentistry, 2018; 10(12), e1161.

[8] Hormzábal, F., López, M., Calderón, N., Leppe, J. \& Castro, A. Nivel de conocimiento sobre conceptos básicos de salud bucal de estudiantes del año de egreso de la carrera de educación parvularia de las universidades de 
la región metropolitana de Chile. Rev. Soc. Chil. Odontopediatría. 2012; Vol. 27(2) 4-13.

[9] Mishra, A., Pandey, R. K., Chopra, H., \& Arora, V. Oral health awareness in schoolgoing children and its significance to parent's education level. Journal of indian society of pedodontics and preventive dentistry. 2018. 36(2), 120.

[10] MINSAL. Chile: MINSAL; 2018 [citado 29 jul 2019]. Capítulo IV: Pastas dentales fluoruradas [pág. 22]. Disponible en: https://diprece.minsal.cl/wpcontent/uploads/2019/02/norma-defluoruros_conResEx_V2019.pdf

\section{CÓMO CITAR ESTE ARTÍCULO}

Chang S, Quiroz M. Evaluación del conocimiento del programa preescolar de salud bucal en parvularias, mediante una encuesta. Appli. Sci. Dent. 2020, 1(1): 17- 25

DOI: 10.22370/asd.2020.1.1.2109 